\title{
High velocity impact on different hybrid architectures of 2D laminated and 3D warp interlock fabric composite
}

\author{
B. Provost ${ }^{1,2}$, F. Boussu ${ }^{1,2}$, D. Coutellier ${ }^{1,3}$, D. Vallee ${ }^{4}$, and F. Rondot ${ }^{5}$ \\ ${ }^{1}$ Univ. Lille Nord de France, 59000 Lille, France \\ ${ }^{2}$ Ensait, Gemtex, 59100 Roubaix, France \\ ${ }^{3}$ Uvhc, Lamih Fre CNRS 3304, 59300 Valenciennes, France \\ ${ }^{4}$ Nexter Systems, Satory, 11 allée des Marronniers, 78022 Versailles, France \\ 5ISL, 5 rue du Général Cassagnou, BP. 70034, 68301 Saint-Louis Cedex, France
}

\begin{abstract}
For decades, conventional amour shield is mainly oriented on metallic materials which are today well-known. Since the use of non conventional threats as IEDs, performances of those protections are required to be upgraded. The expected improvements that manufacturers are looking for are mainly oriented to the weight reduction which is the key parameter to reduce the fuel consumption, increase the payload, and offer more manoeuvrability to vehicles [1].

However, the difficulty is to reduce as cautiously as possible the total mass of the protection solution while ensuring the safety of the vehicle. One of the possible solutions is to use new combinations of materials, able to be more efficient against new threats and lighter than the traditional steel armour.

It is in this context that the combination between some well-known ballistic alloys and textile composite material appear as a high potential solution for armour plated protection. Indeed, used as a backing, textile composite material present some interesting properties such as a very low density compared with steel and good behaviour in term of ballistic efficiency.

This study proposes to test and compare the behaviour and efficiency of three different textile composite backings.
\end{abstract}

\section{Introduction}

Several studies have been made in order to understand the impact behaviour of composite materials as well as improve those textile composite backings, by optimizing the parameters of the fibrous reinforcement or the resin and its process.

Dangers of new treats such as high velocity projectiles have also been identify. Those steel projectiles, accelerated from 1000 to $2000 \mathrm{~m} / \mathrm{s}$, obey to a non conventional impact law, and armour protections need to be adapted in order to be able to resist to those threats.

It has been established that 3D warp interlock fabrics have different mechanical properties than 2D fabric made of the same material [2-5]. Indeed, 3D warp interlock fabric present, due to their unusual bonding between layers, interesting properties during impact.

The difference of behaviour under impact of different types of textile composite backing will be study in order to figure out which one is the more adapted against high speed impact.

Three different textile backings have been tested, a classic 2D laminated composite, a 3D warp interlock with a thermoplastic matrix and a 3D warp interlock with a heathardening matrix.

It was observed that all of them present very different behaviour under impact.

\section{Experimental study}

High speed impact tests have been carry out thanks to powder gun accelerating a $54 \mathrm{~g}$ cylinder shaped steel projectile to high velocity ranging from $1000 \mathrm{~m} / \mathrm{s}$ to over $2000 \mathrm{~m} / \mathrm{s}$.
This projectile impact perpendicularly an armoured shield made of a well know allow metal plate and two textile backings (see figure 1).

The thickness of the metallic part is always constant and the thicknesses of the 2 composite backings have been respectively designed to respect 8 and $5 \mathrm{~mm}$.

Three different textile structures were necessary to create those three hybrid backings.

The first textile structure is a classic 2D laminated composite, made of several pre-impregnated plies made with an aramid yarn of 3360 dTex linear density. (Those composites are widely used in hard ballistic protection). Those plies are assembled under high heat and pressure to obtain the final composite (see figure 2 and 3 ).

The second type of textile structure is a 3D warp interlock fabric composite designed in the GEMTEX laboratory. The main characteristic of those fabrics is their unusual bonding between plies created by the specific evolution of given warp yarns through the fibrous structure. This textile structure was woven with a double yarn in the weft direction, this double yarn is composed of a 3360 dTex aramid yarn and 1400 dTex low density polyethylene yarn. Three plies of this structure were assembled under high heat and pressure in order to form the final composite (see figure 4 and 5).

The third type of textile structure is the same 3D warp interlock fabric introduced previously, but manufactured only with a 3360 dTex aramid yarn. The three plies of this fabric are assembled according to a classic infusion method; the resulting composite is compact under high pressure in order to reach the expected thickness and amount of resin in the final composite. The resin is a heat-hardening matrix: Vinyl-ester (see figure 6 and 7). 


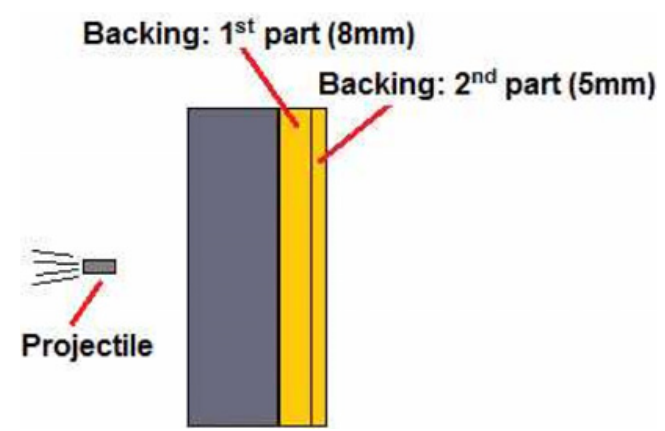

Fig. 1. Test configuration.

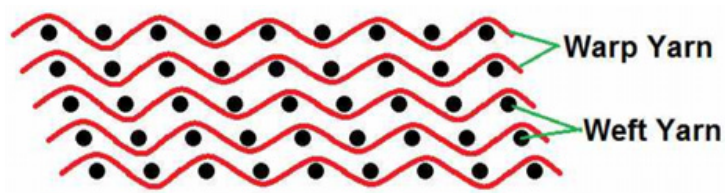

Fig. 2. Cross-sectional view of an assembly of pre-impregnated 2D laminates.

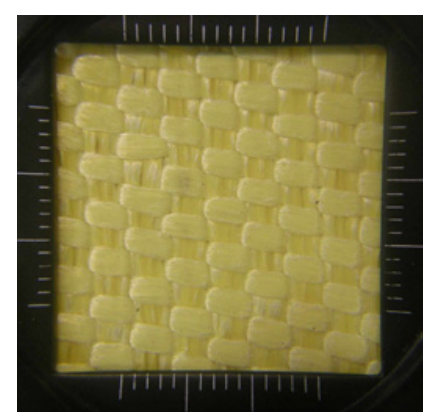

Fig. 3. View of the surface of the pre-impregnated 2D laminates.

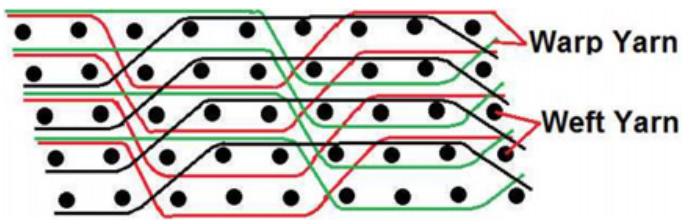

Fig. 4. Cross sectional view of one ply of 3D warp interlock fabric with a thermoplastic matrix.

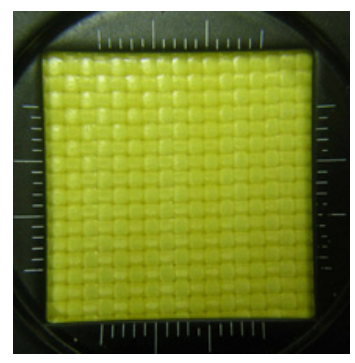

Fig. 5. View of the surface of the 3D warp interlock fabric with a thermoplastic matrix.

From characteristics of those composite that we identify during the previous test campaign as well as during their manufacturing process, we have decided to test them using three different combinations.

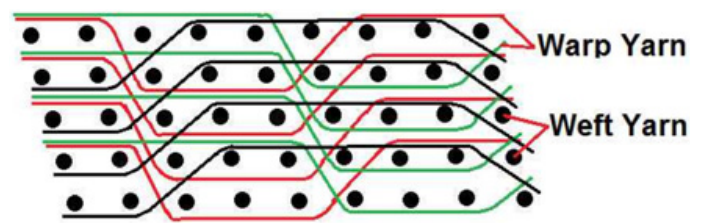

Fig. 6. Cross sectional view of one ply of 3D warp interlock fabric with a heat-hardening matrix.

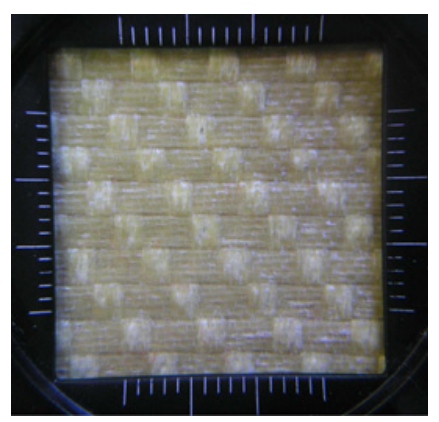

Fig. 7. View of the surface of the 3D warp interlock fabric with a heat-hardening matrix.
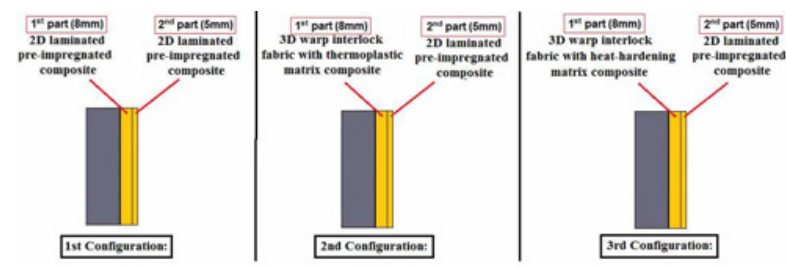

Fig. 8. Schematic view of the 3 tested configurations.
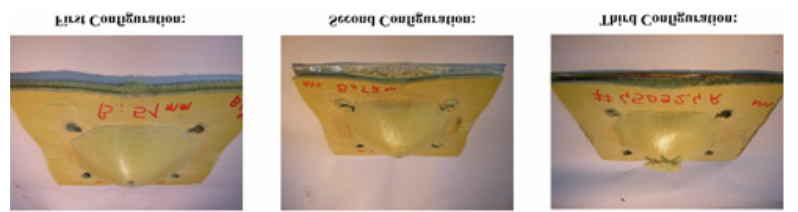

Fig. 9. View of the top surface of the second part $(5 \mathrm{~mm})$ of the backing.

The first configuration of backing is a fully 2D laminates. The first part is $8 \mathrm{~mm}$ of $2 \mathrm{D}$ laminated composite and the second part, $5 \mathrm{~mm}$ of 2D laminated composite.

The second configuration of backing is a combination of $8 \mathrm{~mm}$ of 3D warp interlock composite (thermoplastic matrix) and 5mm of 2D laminated composite.

The third and last configuration is a combination of $8 \mathrm{~mm}$ of 3D warp interlock composite (heat-hardening matrix) and $5 \mathrm{~mm}$ of 2D laminated composite.

Those three configurations are summarised in the figure below (see figure 8).

\section{Experimental results}

Those three configurations were tested in the case of a high velocity impact, at a given speed between $1000 \mathrm{~m} / \mathrm{s}$ and $2000 \mathrm{~m} / \mathrm{s}$. Measurements of the projectile velocity before 


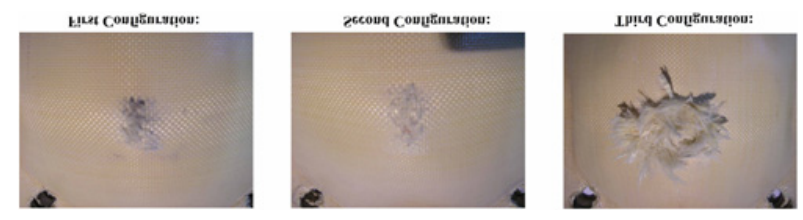

Fig. 10. Detail view of the top surface of the second part $(5 \mathrm{~mm})$ of the backing.
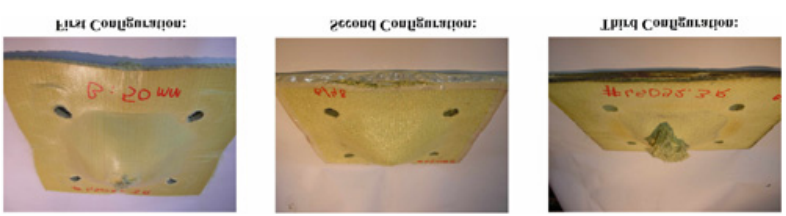

Fig. 11. View of the top surface of the first part $(8 \mathrm{~mm})$ of the backing.

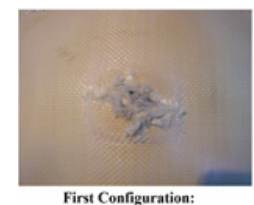

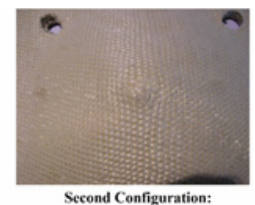

Second Configuration:

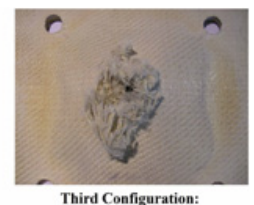

Third Configuration:
Fig. 12. Detail view of the top surface of the first part $(8 \mathrm{~mm})$ of the backing.

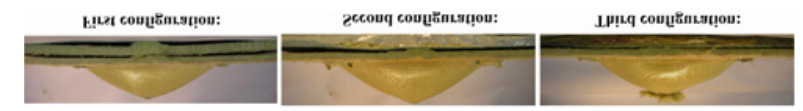

Fig. 13. Side view of the 3 configurations after impact test.

and after impact are performed thanks to several x-ray equipments.

We can observe that those three tests give very different results, especially in term of protection.

1) First configuration:

The first configuration has stopped the projectile. The first part of the backing $(8 \mathrm{~mm})$ shows an important back-face deformation of $48 \mathrm{~mm}$ as well as an important damage area (see figure 11,12). This part is entirely perforated. We can observe on this part of the backing that the warp and weft yarns of the fabric were more stress at the impact point since all the broken yarns in this area under shearing stress form the typical cross-shaped damage area on fabric.

The second part of this backing $(5 \mathrm{~mm})$ presents an important back-face deformation of $49 \mathrm{~mm}$. This part is undamaged even if we can observe the beginning of the tensile fibre breakage on the back-face of the composite due to this important deformation (see figure 9, 10).

2) Second Configuration:

The second configuration also stopped the projectile. The first part of the backing $(8 \mathrm{~mm})$ is partially perforated; which means that the projectile has perforated two of the three layers of the composite structure. The back-face deformation is $48 \mathrm{~mm}$ height (see figure 11, 12).
The second part of the backing remains intact although presenting an important deformation of $57 \mathrm{~mm}$.

We can observe the beginning of tensile fibre breakage on the back-face of the composite due to an important deformation (see figure 9, 10).

3) Third Configuration:

At this impact speed, the third configuration has been perforated. The first part $(8 \mathrm{~mm})$ shows a resulting hole corresponding to the exact size of the projectile which means a pure shearing stress during the impact. An important damage area on the back-face reveals yarns which have been pulled out of the structure. This first part of the backing presents a very small deformation; the maximum back-face deformation is $34 \mathrm{~mm}$ height (see figure 11,12).

The second part of the backing $(5 \mathrm{~mm})$ also presents a small entrance hole while damages occurred at the back-face are still important although more localized. The maximum back-face deformation of the second part is $49 \mathrm{~mm}$ height. The residual speed of the projectile still remains high after the perforation of this protection (see figure 9,10 ).

\section{Conclusion}

This study has revealed that textile composite backings show different behaviours under a same impact according to the different composition of the composite solution.

The first observation is that the third configuration is not adapted to the threat; indeed, we can establish a complete failure of this protection which was fully perforated. This can be attributed to the very stiff behaviour of the 3D warp interlock fabric with the heat-hardening matrix. This important stiffness is due to the vinyl-ester matrix which tends to give to the material a non-elastic and brittle behaviour.

This rigidity is highly inappropriate since it doesn't allow the necessary energy absorption deformation of the backing.

The second observation we can make is the ability of both first and second configuration to stop the projectile. As describe previously, the first configuration, the 2D pre-impregnated laminates of the composite show a first perforated part of $8 \mathrm{~mm}$, while the second part of $5 \mathrm{~mm}$ still remains undamaged. Considering this second configuration; the 3D warp interlock fabric impregnated with a thermoplastic matrix reveals a first part $(8 \mathrm{~mm})$ notfully perforated and an entire un-perforated second part $(5 \mathrm{~mm})$.

This interesting behaviour of the 3D warp interlock fabric with the thermoplastic matrix can be explained by the improved flexibility of the reinforcement. Indeed, the bonding of yarns in the thickness direction coupled with the non systematic bonding of yarns on a same layer tend to give a textile structure more able to be deformed than a similar structure made of $2 \mathrm{D}$ pre-impregnated plies of laminates.

It is possible also to observe the phenomenon of increased elasticity due to the resin comparing the second 
and third configurations together. Both of them present the same textile reinforcement, only the matrix change and give very different results.

The choices of the different backing parameters such as the resin and the structure of the textile reinforcement are crucial in order to reach the expected and safe protection.

In this study, it has been demonstrated that the better impact behaviour is reached by samples using 3D warp interlock fabric impregnated with thermoplastic matrix.

\section{Acknowledgements}

I would like to thanks the ISL for carrying out all the impact tests and NEXTER SYSTEMS for providing advices and funds to conduct this research.

\section{References}

1. Antonio Miravete, 1999, 3-D textile reinforcements in composite materials, WP, ISBN-13: 978-1-85573-3763

2. F Chen and J.M.Hodgkinson, Impact behaviour of composites with different fibre architecture, Proc. IMechE Vol. 223 Part G: J. Aerospace Engineering, JAERO451 DOI: $10.1243 / 09544100 J A E R O 451$

3. Julian HU, 3D fibrous assemblies, properties applications and modelling of three dimensional textile structures, WP number 74, page 105, ISBN 978-1-84569377-0, 2008

4. Ashok Bhatnagar, Lightweight ballistic composite, 2006, WP, ISBN-13: 978-1-85573-941-3

5. F. Boussu X. Legrand and A. Serret, General clustering of warp interlocks structures ITMC 2007 International Conference 